\title{
Adolescentes con trastorno por consumo de sustancias: Una caracterización de personalidad a través de las normas chilenas del MACI*
}

\author{
Eugenia V. Vinet ${ }^{1 a}$, Ximena Faúndez ${ }^{1 b}$, \\ Marcela Larraguibel ${ }^{2}$. \\ Application of Millon Adolescent \\ Clinical Inventory (MACI) \\ to drug abuser adolescents
}

\begin{abstract}
Background: Adolescence is the most vulnerable development stage to start drug abuse in our country. Moreover, adolescent personality may be an individual risk factor for drug abuse. Aim: To characterize the personality of adolescent drug abusers using the Millon Adolescent Clinical Inventory (MACI) and its Chilean norms. Patients and methods: Adolescents consulting in a mental health facility were studied. MACI was applied to 46 subjects, aged $16 \pm 1$ years (30 males) with a history of drug abuse and compared with 58 peers aged $15 \pm 2$ years ( 34 males) without such history. Results: Male and female drug abuser adolescents had a transgressor type of personality. Males had a high degree of sociability and unrestrained sexuality while females present signs of sexual abuse, suicide risk, and a more severe personality disorder. Conclusions: The personality traits detected in this group of drug abuser adolescents using Chilean norms is similar to those detected abroad and should help to plan rehabilitation (Rev Méd Chile 2009; 137: 466-74).
\end{abstract}

(Key words: Adolescent; Personality disorders; Substance-related disorders)

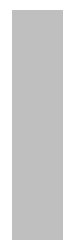

Recibido el 17 de julio, 2008. Aceptado el 24 de diciembre, 2008.

Estudio financiado por la Dirección de Investigación de la Universidad de La Frontera a través del Proyecto DIUFRO 120612.

${ }^{1}$ Departamento de Psicología, Universidad de La Frontera. Temuco, Chile. ${ }^{2}$ Departamento de Psiquiatría y Salud Mental, Universidad de Chile. Santiago de Chile.

aPsicóloga, Doctora en Psicología Clínica y de la Salud

bPsicóloga, Magíster en Psicología

Correspondencia a: Eugenia V. Vinet. Departamento de Psicología, Universidad de La Frontera; Casilla 54- D. Temuco, Chile. Fax: (45) 341480, E mail: evinet@ufro.cl

*Este artículo presenta resultados parciales de la Tesis Doctoral de la primera autora. 
$\mathrm{E}^{1}$ alto consumo de drogas y los problemas sociales derivados han hecho que éste sea uno de los problemas de salud más importantes que enfrenta la sociedad mundial en nuestra época. Se estima que unos 200 millones de personas, $5 \%$ de la población mundial de 15 a 64 años de edad, han consumido drogas ilícitas al menos una vez en el último año ${ }^{1}$.

En Chile, el último informe del Consejo Nacional para el Control de Estupefacientes (CONACE) en población general señala estabilidad en las prevalencias de uso de drogas con respecto a los informes previos, excepto para el consumo de marihuana que ha aumentado en los últimos años afectando a personas cada vez más jóvenes; además, señala a la adolescencia como la etapa más vulnerable para el inicio del consumo de drogas ${ }^{2}$.

El último informe en población escolar desarrollado con alumnos de Octavo Básico a Cuarto Medio en el año 2007, registra prevalencias de $35 \%$ para el consumo de tabaco, 38\% para el alcohol, 4\% para la cocaína y $15,7 \%$ para la marihuana, observándose claras diferencias según sexo con valores menores para las mujeres ${ }^{3}$.

Estos estudios indican que en población escolar existe una baja percepción de riesgo para la marihuana y una percepción de facilidad en el acceso y disponibilidad para las drogas en general. Además, el informe en población escolar destaca la relación entre la marihuana y trastornos graves de la adolescencia pues su uso duplica el riesgo de tener problemas con la policía, incrementa el riesgo de agresión y victimización sexual y aparece relacionada con impulsos autodestructivos $^{3}$. Sin embargo, este informe no entrega información sobre comorbilidad entre consumo de drogas y conducta antisocial, desórdenes psiquiátricos y trastornos de la personalidad en adolescentes, la cual suele reportarse en estudios internacionales ${ }^{4-6}$.

Una visión multidimensional de los problemas de consumo debe incluir a las características de personalidad como un factor de riesgo individual. Sin embargo, en nuestro país existen pocos estudios sobre personalidad adolescente y consumo.

A nivel internacional, la personalidad ha sido considerada en numerosos estudios sobre consumo de drogas, utilizándose diversos instrumentos de evaluación para caracterizar a los adolescentes consumidores; entre ellos destaca el Inventario Clínico para Adolescentes de Millon, $\mathrm{MACI}^{7}$, el cual posee una versión chilena ${ }^{8}$, es de fácil aplicación y ha presentado alta confiabilidad y validez en nuestro país 9 .

El MACI es un autoinforme de construcción racional, que sigue los modelos de aprendizaje biosocial y evolutivo de la personalidad desarrollados por Millon ${ }^{10,11}$ y evalúa estilos de personalidad, preocupaciones psicológicas y psicopatología en adolescentes. Los estilos de personalidad dimensionan funcionamientos psicológicos relativamente estables que surgen a través del desarrollo infantil y se estabilizan en la adolescencia como precursores de los estilos de personalidad adulta. Las preocupaciones psicológicas representan aspectos significativos del contexto de desarmollo del adolescente que pueden causarle preocupación, incluyen aspectos del desarrollo de la identidad, la corporalidad, la sexualidad y las relaciones sociales con los pares, la familia y la sociedad mayor. El área psicopatológica incluye los trastornos afectivos y de la adaptación social que tienen mayor prevalencia en la adolescencia, entre los que se destaca la tendencia al uso de sustan$\operatorname{cias}^{7,9}$.

Diversos estudios han utilizado el MACI para caracterizar a adolescentes consumidores de sustancias. En Estados Unidos de Norteamérica se encontró que los adolescentes diagnosticados con trastornos por consumo de sustancias (TCS) se diferenciaban de los adolescentes diagnosticados con otro tipo de trastornos en 14 de las 27 escalas clínicas, destacando puntuaciones significativamente más altas en las escalas Trasgresor, Insensibilidad Social, Predisposición a la Delincuencia y Tendencia al Abuso de Sustancias y significativamente más bajas en Sentimientos Ansiosos $^{12}$. En Chile se encontraron diferencias significativas, expresadas en puntajes directos, entre una muestra de adolescentes varones consumidores de drogas y una muestra de escolares sin antecedentes de consumo en todas las escalas clínicas del MACI con excepción de Insensibilidad Social, las diferencias más importantes fueron la elevación de los puntajes de los consumidores en la escala Trasgresor y el decremento en la escala Conformista (Tesis de Licenciatura en Psicología de A. Alarcón, X. Faúndez, C. 
Hita y P. Rodríguez. Universidad de La Frontera, Chile, 2004).

Esta tesis distinguió en los consumidores cuatro tipos diferenciales obtenidos a través de un análisis de conglomerados de las escalas de Patrones de la Personalidad. Ellos fueron denominados según sus características más relevantes como Inhibido-Emocionalmente, OposicionistaTrasgresor, Subclínico y Egoísta-Dramatizador. Todos compartieron elevaciones significativas en el patrón de personalidad Trasgresor y la Tendencia al Abuso de Sustancias, sin embargo fue el tipo Oposicionista-Trasgresor el que reunió el mayor número de casos con abuso y dependencia de drogas $^{13}$.

El objetivo del presente estudio es caracterizar, a través del MACI, a adolescentes de ambos sexos consultantes con TCS utilizando las normas chilenas para el $\mathrm{MACI}^{14}$, siendo un objetivo adicional del estudio el verificar el comportamiento de esta nueva escala normativa recientemente desarrollada.

\section{PACIENTES Y MÉTODO}

La muestra, constituida por 104 adolescentes de 13 a 19 años consultantes en Servicios de Salud Mental, incluyó a 30 varones y 16 mujeres con abuso o dependencia de sustancias y, como contraparte, un grupo de 34 varones y 24 mujeres adolescentes, consultantes de los mismos servicios, sin TCS.

Todos los participantes fueron voluntarios, firmaron un consentimiento informado, sostuvieron una entrevista clínica semiestructurada y contestaron el MACI en grupos pequeños. La entrevista clínica siguió un protocolo de construcción ad-hoc ${ }^{13}$ que aportó información sobre motivos de consulta, características familiares y sintomatología; además, en los consumidores se indagó sobre las características y el tipo de consumo. Este fue clasificado según las categorías CONACE para el consumo no problemático (experimental, ocasional, habitual) y según criterios del DSM-IV para los TCS (abuso y dependencia).

La caracterización psicológica de los jóvenes se realizó con el MACI. Este consta de 160 ítems con formato verdadero-falso organizados en 31 escalas,
27 de ellas con significado clínico; estas son doce escalas de Patrones de Personalidad, ocho escalas de Preocupaciones Expresadas y siete escalas de Síndromes Clínicos. Las escalas no clínicas son tres escalas de control y una escala de validez. Sus denominaciones, según la versión chilena, aparecen en la primera columna de las Tablas 3 y 4 .

$\mathrm{Su}$ fiabilidad en muestras chilenas es similar a la obtenida por Millon ${ }^{7}$ y oscila entre coeficientes Alfa de Cronbach de .54 (D) y .90 (8B) en adolescentes no-pacientes y entre .51 (D) y .91 (8B y B) en adolescentes con problemas psicológicos, con medianas de .79 y .81, respectivamente ${ }^{9}$. El MACI posee en Chile una adecuada capacidad para discriminación entre adolescentes normales y con problemas psicológicos ${ }^{9}$ y es un instrumento idóneo para caracterizar psicológicamente a grupos de adolescentes con problemas clínicos ${ }^{14}$ y de desadaptación social ${ }^{15}$.

El MACI posee tres grupos de normas. Las dos primeras (estadounidenses y españolas) están expresadas en puntajes de tasa base asociados a tasas de prevalencia de trastornos mentales; el tercer grupo, normas chilenas expresadas en puntajes transformados con puntos de corte fueron desarrolladas recientemente ${ }^{14}$.

Las normas chilenas incluyen: (a) baremos diferenciados por sexo para adolescentes entre 13 y 19 años, (b) una evaluación de la capacidad de discriminación de las escalas entre sujetos de población general y sujetos clínicos y (c) una evaluación de la pertinencia cultural de las escalas. La conjunción de estas dos evaluaciones clasifica a las escalas del MACI en escalas diferenciadoras Acordes con la Teoría (AT), escalas diferenciadoras Contra-Teóricas (CT) y escalas No Diferenciadoras (ND). Estas normas se expresan en una escala de 100 puntos que aúna criterios dimensionales y categoriales para la evaluación de las características medidas. Su puntaje central (PT 50) está asociado al punto de corte que, en cada escala, diferencia entre funcionamiento sano y funcionamiento alterado o patológico ${ }^{14}$.

El MACI fue aplicado según sus instrucciones estándares ${ }^{7}$, los protocolos fueron codificados en puntajes directos y luego convertidos a Puntajes Trasformados con Puntos de Corte según las normas chilenas ${ }^{14}$. Las puntuaciones obtenidas en los adolescentes con y sin TCS fueron comparadas mediante pruebas $t$ de Student. 


\section{RESULTADOs}

Caracterización general. Los consultantes con TCS tuvieron una edad media de 16,4 años (DS $=1,29$ ) y una media de 9,3 años de educación (DS $=1,74)$. Los consultantes sin TCS tuvieron una edad media de 15,3 años (DS $=1,91$ ) y una media de 9,4 años de educación (DS =1,77). La mayor edad con similar nivel educacional de los adolescentes con TCS evidencia que ellos suelen presentar dificultades escolares con respecto a sus pares $\sin$ TCS $(t=3,68, p<0,001)$.

El motivo de consulta principal de los adolescentes con TCS difiere, en la mayoría de los casos, del motivo de consulta de los adolescentes sin TCS (Tabla 1). En los hombres con TCS, predomina el uso de sustancias (60\%) y los problemas externalizados (30\%), en tanto que en las mujeres con TCS predomina el uso de sustancias y la conducta autodestructiva (25\% en cada caso).

Con respecto al consumo, todos los consumidores, hombres y mujeres, presentaron policonsumo. El 40\% de los hombres y 31\% de las mujeres recibió el diagnóstico de abuso de al menos una sustancia; el resto recibió el diagnóstico de dependencia, también de al menos una sustancia. La Tabla 2 muestra las sustancias consumidas en la última semana y el diagnóstico de consumo por sustancia.

Tabla 1. Motivo de consulta principal de 104 adolescentes con y sin trastorno por consumo de sustancias (TC S)

\begin{tabular}{|c|c|c|c|c|}
\hline \multirow[t]{2}{*}{$\begin{array}{l}\text { Motivo de consulta } \\
\text { principal }\end{array}$} & \multicolumn{2}{|c|}{ H ombres $(n=64)$} & \multicolumn{2}{|c|}{ M ujeres $(n=40)$} \\
\hline & $\begin{array}{l}\text { Con TCS } \\
(n=30)\end{array}$ & $\begin{array}{l}\operatorname{Sin} \text { TCS } \\
(n=34)\end{array}$ & $\begin{array}{l}\text { Con TCS } \\
(n=16)\end{array}$ & $\begin{array}{l}\text { Sin TCS } \\
(n=24)\end{array}$ \\
\hline Problemas internalizados & 3,3 & 32,4 & 18,8 & 41,7 \\
\hline Problemas escolares & 3,3 & 23,5 & 0,0 & 12,5 \\
\hline Problemas externalizados & 30,0 & 20,6 & 18,8 & 16,7 \\
\hline Conducta autodestructiva & 0,0 & 0,0 & 25,0 & 8,3 \\
\hline Uso de sustancias & 60,0 & 0,0 & 25,0 & 0,0 \\
\hline Inhabilidad social & 0,0 & 5,9 & 0,0 & 4,2 \\
\hline Disfunción familiar & 3,3 & 17,6 & 12,5 & 16,7 \\
\hline
\end{tabular}

Nota. Valores expresados en porcentajes sobre el total de cada columna.

Tabla 2. Reporte de consumo de la última semana y diagnóstico de consumo por sustancia en hombres y mujeres con TCS

\begin{tabular}{|c|c|c|c|c|c|c|}
\hline \multirow[t]{2}{*}{ Sustancia } & \multicolumn{3}{|c|}{ H ombres $(n=30)$} & \multicolumn{3}{|c|}{ Mujeres $(n=16)$} \\
\hline & $\begin{array}{l}\text { Consumo en } \\
\text { última semana }\end{array}$ & Abuso & D ependencia & $\begin{array}{l}\text { Consumo en } \\
\text { última semana }\end{array}$ & Abuso & Dependencia \\
\hline Cigarrillos & 90,0 & 10,0 & 6,7 & 93,8 & 6,3 & 31,3 \\
\hline Alcohol & 40,0 & 40,0 & 13,3 & 18,8 & 31,3 & 25,0 \\
\hline Marihuana & 46,0 & 50,0 & 43,3 & 12,5 & 25,0 & 25,0 \\
\hline Pasta base & 10,0 & 10,0 & 6,7 & - & 12,5 & - \\
\hline Cocaína & 3,3 & 16,7 & 3,3 & 6,3 & 6,3 & - \\
\hline Solventes & - & 16,7 & - & - & 6,3 & 6,3 \\
\hline Otros & 20,0 & 13,4 & 6,6 & 6,3 & 6,3 & 12,5 \\
\hline
\end{tabular}

Nota. Valores expresados en porcentajes sobre el total de hombres o mujeres. 
Tabla 3. Estadísticos descriptivos del M ACI y contrastes según presencia 0 ausencia de TCS en 64 consultantes varones

\begin{tabular}{|c|c|c|c|c|c|c|}
\hline & \multirow[t]{2}{*}{ Escalas C línicas } & \multicolumn{2}{|c|}{$\begin{array}{c}\text { Con TCS } \\
(n=30)\end{array}$} & \multicolumn{2}{|c|}{$\begin{array}{l}\text { Sin TCS } \\
(n=34)\end{array}$} & \multirow[t]{2}{*}{$\mathbf{t}$} \\
\hline & & M & DT & M & DT & \\
\hline 1 & Introvertido & 47,67 & 15,78 & 52,97 & 12,98 & $-1,46$ \\
\hline $2 \mathrm{~A}$ & Inhibido & 46,77 & 16,86 & 53,48 & 14,29 & $-1,71$ \\
\hline $2 B$ & Afligido & 53,33 & 17,09 & 55,87 & 15,17 & $-0,62$ \\
\hline 3 & Sumiso & 61,37 & 9,98 & 46,85 & 11,94 & $5,24^{* 1 * k}$ \\
\hline 4 & Dramatizador & 44,50 & 14,02 & 54,09 & 14,28 & $-2,70^{* *}$ \\
\hline 5 & Egoísta & 48,90 & 10,47 & 52,41 & 15,41 & $-1,08$ \\
\hline $6 \mathrm{~A}$ & Trasgresor & 69,23 & 9,65 & 50,12 & 14,17 & $6,22^{* \text { **x }}$ \\
\hline $6 \mathrm{~B}$ & Poderoso & 62,67 & 10,31 & 47,32 & 20,14 & 3,90 **** \\
\hline 7 & Conformista & 62,00 & 8,52 & 49,24 & 16,15 & 3,97 **1* \\
\hline $8 \mathrm{~A}$ & Oposicionista & 58,20 & 7,44 & 51,97 & 14,15 & $2,22^{*}$ \\
\hline $8 B$ & Autodegradante & 53,37 & 14,60 & 53,03 & 17,77 & 0,08 \\
\hline 9 & Tendencia límite & 58,33 & 13,88 & 53,15 & 21,68 & 1,15 \\
\hline $\mathrm{A}$ & Difusión de la identidad & 55,63 & 14,03 & 51,68 & 13,83 & 1,14 \\
\hline B & Autodevaluación & 53,67 & 16,78 & 57,03 & 17,89 & $-0,77$ \\
\hline $\mathrm{C}$ & Desaprobación corporal & 36,67 & 22,58 & 46,00 & 27,53 & $-1,47$ \\
\hline $\mathrm{D}$ & Incomodidad sexual & 57,40 & 11,98 & 47,74 & 10,22 & $3,48^{* *}$ \\
\hline $\mathrm{E}$ & Inseguridad grupal & 45,63 & 21,83 & 51,62 & 16,18 & $-1,26$ \\
\hline $\mathrm{F}$ & Insensibilidad social & 59,60 & 8,95 & 48,91 & 12,13 & $3,95^{\text {*1* }}$ \\
\hline $\mathrm{G}$ & Discordia familiar & 63,67 & 10,60 & 52,03 & 16,22 & $3,43^{\text {*ax }}$ \\
\hline $\mathrm{H}$ & Abuso infantil & 55,03 & 12,67 & 51,09 & 14,48 & 1,15 \\
\hline $\mathrm{AA}$ & Disfunciones alimentarias & 37,60 & 21,91 & 44,34 & 25,40 & $-1,12$ \\
\hline BB & Tendencia abuso sustancias & 73,00 & 7,91 & 45,64 & 14,64 & $9,34^{\text {**1* }}$ \\
\hline $\mathrm{CC}$ & Predisposición delincuencia & 69,50 & 11,47 & 45,44 & 13,79 & $7,53^{*}$ *1* \\
\hline $\mathrm{DD}$ & Tendencia a la impulsividad & 67,40 & 11,56 & 54,58 & 18,48 & $3,33^{\text {** }}$ \\
\hline $\mathrm{EE}$ & Sentimientos de ansiedad & 65,80 & 10,79 & 46,21 & 11,88 & $6,87^{*}$ *小⿰㇇⿰亅⿱丿丶丶 \\
\hline FF & Afecto depresivo & 44,50 & 19,13 & 53,03 & 17,30 & $-1,87$ \\
\hline GG & Tendencia suicida & 50,83 & 17,32 & 49,76 & 22,03 & 0,21 \\
\hline
\end{tabular}

***p $<0,001 *$ * $<<0,01 * p<0,05$.

Caracterización según el MACI. La comparación entre adolescentes con TCS y sin TCS, conforme a las normas chilenas, se presenta por separado según sexo.

Para los hombres, la Tabla 3 muestra diferencias significativas en 13 de las 27 escalas clínicas abarcando Patrones de Personalidad, Preocupaciones Expresadas y Síndromes Clínicos. En el área de patrones, los hombres con TCS presentan elevaciones significativas en las escalas Sumiso, Trasgresor, Poderoso, Conformista y Oposicionista; dado que Sumiso y Conformista son escalas contra-teóricas, sus elevaciones deben ser inter- pretadas como no-sumisión y no-conformismo ${ }^{14}$. En el área de preocupaciones, la elevación más significativa está en Insensibilidad Social y es acompañada de elevaciones en Discordia Familiar e Incomodidad Sexual; esta última es una escala contra-teórica y debe interpretarse como desinhibición de la sexualidad. Las elevaciones en Síndromes Clínicos incluyen tendencia al abuso de sustancias, predisposición a la delincuencia, sentimientos de ansiedad y tendencia a la impulsividad; para su interpretación se debe considerar que la escala Sentimientos de Ansiedad es contrateórica en hombres y que su elevación debe 
Tabla 4. Estadísticos descriptivos del M ACI y contraste según presencia o ausencia de TCS en $\mathbf{4 0}$ consultantes mujeres

\begin{tabular}{|c|c|c|c|c|c|c|}
\hline & \multirow[t]{2}{*}{ Escalas } & \multicolumn{2}{|c|}{$\begin{array}{l}\text { Con TCS } \\
(n=16)\end{array}$} & \multicolumn{2}{|c|}{$\begin{array}{l}\text { Sin TCS } \\
(n=24)\end{array}$} & \multirow[t]{2}{*}{$\mathbf{t}$} \\
\hline & & $M$ & DT & $M$ & DT & \\
\hline 1 & Introvertido & 59,25 & 15,16 & 58,58 & 15,93 & 0,13 \\
\hline $2 \mathrm{~A}$ & Inhibido & 61,63 & 14,24 & 60,29 & 16,58 & 0,26 \\
\hline $2 B$ & Afligido & 74,13 & 17,77 & 67,87 & 20,67 & 0,98 \\
\hline 3 & Sumiso & 62,69 & 11,56 & 46,83 & 16,43 & $3,34^{\text {** }}$ \\
\hline 4 & Dramatizador & 57,56 & 15,19 & 58,25 & 13,69 & $-0,15$ \\
\hline 5 & Egoísta & 57,88 & 14,05 & 57,61 & 11,52 & 0,07 \\
\hline $6 \mathrm{~A}$ & Trasgresor & 70,25 & 9,46 & 51,87 & 14,92 & $4,35^{\text {**** }}$ \\
\hline $6 \mathrm{~B}$ & Poderoso & 69,44 & 12,22 & 50,92 & 19,42 & $3,70 * *$ \\
\hline 7 & Conformista & 71,06 & 11,86 & 54,62 & 18,93 & 3,09 *** \\
\hline $8 \mathrm{~A}$ & Oposicionista & 71,63 & 13,08 & 56,83 & 16,22 & $3,04 * *$ \\
\hline $8 \mathrm{~B}$ & Autodegradante & 68,38 & 19,36 & 65,77 & 14,63 & 0,47 \\
\hline 9 & Tendencia límite & 74,19 & 17,76 & 64,83 & 23,11 & 1,37 \\
\hline $\mathrm{A}$ & Difusión de la identidad & 64,75 & 17,88 & 60,42 & 17,54 & 0,76 \\
\hline B & Autodevaluación & 67,56 & 22,92 & 67,00 & 16,67 & 0,09 \\
\hline $\mathrm{C}$ & Desaprobación corporal & 63,37 & 28,55 & 56,50 & 27,49 & 0,76 \\
\hline $\mathrm{D}$ & Incomodidad sexual & 55,75 & 7,88 & 51,61 & 12,62 & 1,16 \\
\hline $\mathrm{E}$ & Inseguridad grupal & 57,06 & 18,50 & 56,25 & 20,19 & 0,13 \\
\hline $\bar{G}$ & Discordia familiar & 69,44 & 15,08 & 54,67 & 18,59 & $2,65^{*}$ \\
\hline $\mathrm{H}$ & Abuso infantil & 73,19 & 17,75 & 57,08 & 20,54 & $2,56^{*}$ \\
\hline $\mathrm{AA}$ & Disfunciones alimentarias & 59,00 & 25,40 & 57,38 & 24,63 & 0,20 \\
\hline $\mathrm{BB}$ & Tendencia abuso sustancias & 75,44 & 12,35 & 49,26 & 19,06 & $4,82^{* * *}$ \\
\hline DD & Tendencia a la impulsividad & 72,50 & 11,79 & 53,83 & 18,82 & $3,86^{* * *}$ \\
\hline FF & Afecto depresivo & 63,06 & 21,67 & 61,58 & 22,49 & 0,21 \\
\hline \multirow[t]{2}{*}{ GG } & Tendencia suicida & 78,19 & 16,01 & 61,92 & 23,94 & $2,38 *$ \\
\hline & Escalas No Diferenciadoras & & $\mathrm{Md}_{\mathrm{CC}}$ & \multicolumn{2}{|c|}{$\mathrm{Md}_{\mathrm{SC}}$} & $\mathrm{Z}$ \\
\hline $\mathrm{F}$ & Insensibilidad social & & 81,00 & \multicolumn{2}{|c|}{56,00} & $-2,12 *$ \\
\hline $\mathrm{CC}$ & Predisposición delincuencia & & 95,50 & \multicolumn{2}{|c|}{31,00} & $-4,55^{*}$ * \\
\hline $\mathrm{EE}$ & Sentimientos de ansiedad & & 3,00 & \multicolumn{2}{|c|}{59,00} & $-4,22 * 4 *$ \\
\hline
\end{tabular}

***p $<0,001 *$ * $p<0,01 * p<0,05$.

interpretarse como carencia de manifestaciones ansiosas $^{14}$.

La Figura 1 muestra gráficamente los aspectos más relevantes de la caracterización de los varones con TCS evidenciando que comparten características con el grupo normativo de no-consultantes y con los consultantes sin TCS. El gráfico destaca las elevaciones más marcadas del ámbito de la personalidad (escalas 3, 6A, 6B y 7, No-sumiso, Trasgresor, Poderoso y No-conformista), de las preocupaciones (D, F y G; No-incomodidad Sexual, Insensibilidad Social y Discordia Familiar) y los Síndromes Clínicos más prevalentes (BB, CC, DD y EE; Abuso de Sustancias, Predisposición a la Delincuencia, Tendencia a la Impulsividad y Noansiedad).

La Tabla 4 muestra los resultados de las mujeres. Esta tabla presenta al final y en percentiles los resultados de las escalas no diferenciadoras (Insensibilidad Social, Predisposición a la Delincuencia y 


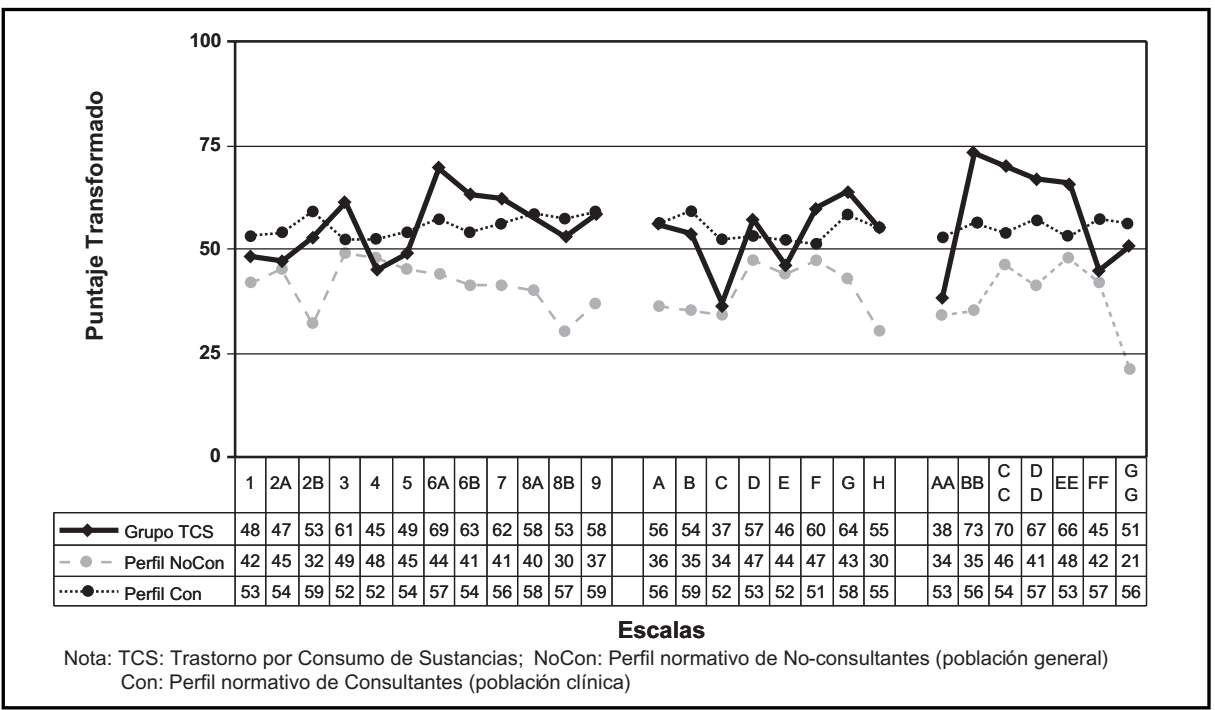

Figura 1. Perfil grupal del MACI de 30 varones con TCS (abuso o dependencia de drogas).

Sentimientos de Ansiedad). Las mujeres consultantes con TCS presentan diferencias significativas con respecto a las consultantes sin TCS en 13 de las 27 escalas clínicas del MACI. En Patrones de la Personalidad las adolescentes con TCS presentan elevaciones en las escalas contra-teóricas Sumiso y Conformista, y en las escalas acordes con la teoría Trasgresor, Poderoso y Oposicionista. En Preocupaciones Expresadas presentan elevaciones en Insen- sibilidad Social, Discordia Familiar y Abuso Infantil. En Síndromes Clínicos presentan elevaciones en Tendencia al Abuso de Sustancias, Predisposición a la Delincuencia, Tendencia a la Impulsividad y Tendencia Suicida y un decremento importante en Sentimientos de Ansiedad.

En el perfil de la Figura 2 se aprecia que las mujeres con TCS tienen una condición clínica grave pues sus puntuaciones están ubicadas, en la

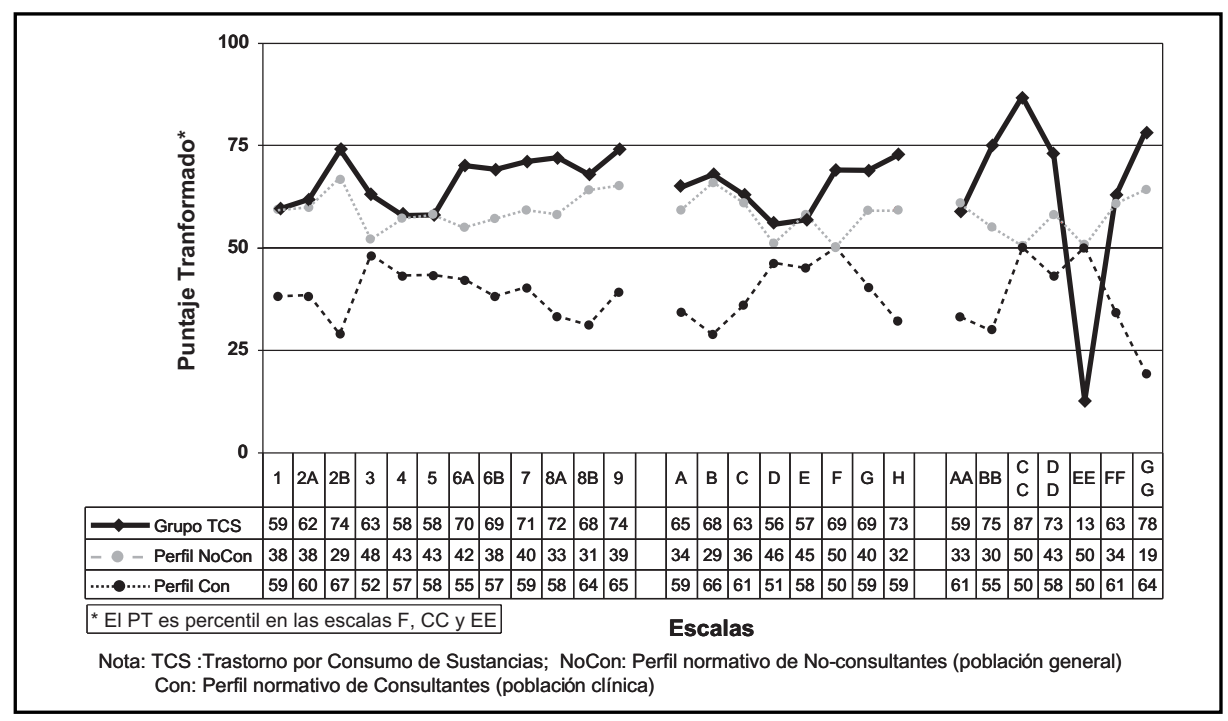

Figura 2. Perfil grupal del MACI de 16 mujeres con TCS (abuso o dependencia de drogas). 
gran mayoría de las escalas, encima de las puntuaciones normativas del grupo clínico, sin observarse puntuaciones similares a las del grupo normal. Además, es importante señalar que el decremento en la escala de ansiedad representa, en este perfil, ausencia de sentimientos ansiosos pues esta escala, expresada en percentiles, no tiene el comportamiento contra-teórico que presenta en los varones ${ }^{14}$.

\section{DisCUSIÓN}

Los principales resultados de este estudio permiten afirmar que el MACI discrimina adecuadamente entre adolescentes con TCS y adolescentes consultantes sin TCS, evidenciando, en varones y mujeres, diferencias significativas en 13 de las 27 escalas clínicas del MACI.

La interpretación de los perfiles de personalidad posibilita la caracterización psicológica de estos adolescentes. Los adolescentes hombres con TCS son irrespetuosos y desconsiderados con los otros, poco tolerantes de las normas, oposicionistas, poco cooperativos y manipuladores de las relaciones interpersonales en función de sus propios intereses. Sin embargo, ellos poseen características de sociabilidad y seducción interpersonal similares a las de los adolescentes normales (decremento en la escala Dramatizador), diferenciándose de los consultantes sin TCS que son más inhibidos y con menos habilidades sociales. En sus Preocupaciones, ellos aparecen como poco sensibles, con problemas familiares y con una sexualidad más desinhibida que lo esperado en la sociedad chilena ${ }^{16}$. En Síndromes Clínicos las elevaciones dan cuenta del trastorno adictivo y son congruentes con lo descrito por la literatura especialmente si se considera que la escala de ansiedad es contra-teórica y debe interpretarse como disminución de la ansiedad naturalmente esperada en la adolescencia.

Las adolescentes mujeres con TCS presentan un perfil de personalidad similar al de los varones consumidores en los tres ámbitos de evaluación del MACI (Patrones de la Personalidad, Preocupaciones Expresadas y Síndromes Clínicos). Sin embargo, se observan elevaciones adicionales en
Abuso Infantil y Tendencia Suicida al compararlas con los varones con TCS indicando que las adolescentes consumidoras tienen una condición clínica claramente más grave.

Las características y perfiles del MACI encontrados en los adolescentes con TCS coinciden con la descripción realizada en estudios estadounidenses $^{12}$ y con los estudios chilenos expresados con puntuaciones directas ${ }^{13}$. Además, los perfiles de hombres y mujeres descritos en el presente estudio se asemejan al tipo Oposicionista-Trasgresor que agrupaba, en los estudios nacionales, al mayor número de adolescentes con consumo problemático de sustancias ${ }^{13}$.

Como información adicional, en el ámbito de las conductas personales de riesgo, se perfila la desinhibición sexual de los varones con TCS (escala D o Incomodidad Sexual del MACI, contrateórica) como un elemento inquietante pero compatible con lo reportado en la literatura internacional ${ }^{17-19}$ y por los terapeutas de este tipo de adolescentes.

Por otra parte, la relación entre Abuso de Sustancias, Abuso Infantil y Tendencia Suicida presente en el perfil de las mujeres consumidoras, ha sido descrita anteriormente a través del concepto "personalidad límite en statu nascen di" 20 el cual representa apropiadamente la gravedad del cuadro clínico de las adolescentes mujeres con TCS.

Finalmente, los resultados indican que las normas chilenas del MACI ${ }^{14}$ permiten caracterizar adecuadamente a los adolescentes consumidores de drogas coincidiendo con las descripciones realizadas en la literatura internacional y nacional. Así, este estudio ofrece una nueva herramienta para el diagnóstico y la elaboración de estrategias de intervención para adolescentes afectados por consumo de sustancias en nuestro país.

\section{Agradecimientos}

Las autoras agradecen la autorización del Comité de Ética del Servicio de Salud Araucanía Sur para realizar este estudio en centros de atención de adolescentes bajo su responsabilidad. Además, agradecen el financiamiento otorgado por la Dirección de Investigación de la Universidad de La Frontera a través del proyecto 120612 . 


\section{REFERENCIAS}

1. United Nations Office on Drugs and Crime. Reporte Anual 2008. Disponible en: http://www.unodc.org/ documents/about-unodc/AR08 WEB.pdf [Consultado el 30 de septiembre de 2008].

2. Comisión Nacional de Control de Estupefacientes. Séptimo estudio nacional de drogas en población general de Chile, 2006. Gobierno de Chile; 2007. Disponible en: http://www.conace.cl/inicio/pdf/ bd928b266121a764e5ea61e6e6ab2ba3.pdf. [Consultado el 20 de noviembre de 2007].

3. Comisión Nacional de Control de Estupefacientes. Séptimo estudio nacional de drogas en población escolar de Chile, 2007. Gobierno de Chile; 2008. Disponible en: http://www.conace.cl/inicio/pdf/ informe ejecutivo Estudio Escolares Conace2007 16-06-2008.pdf. [Consultado el 20 de junio de 2008]

4. Muñoz-Rivas MJ, Graña Gómez JL, Peña Fernández ME, Andreu Rodríguez JM. Influencia de la conducta antisocial en el consumo de drogas ilegales en población adolescente. Adicciones 2002; 14: 313-20.

5. Gerra G, Angioni L, Zaimovic A, Moi G, Bussandri M, BERTACCA S ET AL. Substance use among high-school students: Relationships with temperament, personality traits, and parental care perception. Subst Use Misuse 2004; 39: 345-67.

6. Llorens A, Palmer A, Perewó M. Características de personalidad en adolescentes como predictores de la conducta de consumo de sustancias psicoactivas. Trastornos Adictivos 2006; 7: 90-6.

7. Miшon T. Manual of Millon Adolescent Clinical Inventory. Minneapolis: Nacional Computer Systems; 1993.

8. Vinet E, Brió C, Correa P, Díaz P, Diez M, Echeverría M, Salazar D, Vargas A. (1999). MACI. Traducción y adaptación chilena para uso exclusivo en investigación. Proyecto DIDUFRO 9966. Universidad de La Frontera, Temuco, Chile.
9. Vinet E, Alarcón P. El Inventario Clínico para Adolescentes de Millon (MACI) en la evaluación de adolescentes chilenos. Psykhe 2003; 12: 39-55.

10. MiLon T. Modern psychopathology: A biosocial approach to maladaptative learning and functioning. Philadelphia: Saunders; 1969.

11. MiLON T. Toward a new personology. An evolutionary model. New York: Wiley; 1990.

12. Grilo C, Fehon D, Walker M, Martino S. A comparison of adolescents' inpatients with and without substance abuse using the Millon Adolescent Clinical Inventory. J Youth Adolesc 1996; 25: 379-89.

13. FaÚNDEZ X, VinET E. Tipología empírica de adolescentes consumidores de drogas según MACI. Psykhe, 2009 (en prensa).

14. VINET E, ForUS M. Normas chilenas para el MACI: una integración de criterios categoriales y dimensionadas. Terapia Psicológica 2008; 26: 151-63.

15. Alarcón P, Vinet E, Salvo S. Estilos de personalidad y desadaptación social durante la adolescencia. Psykhe 2005, 14: 3-16.

16. Tironi E, Aristía T, Faverio F. (2005). Comunidad, familia y nación en el Bicentenario: El sueño chileno. Santiago de Chile: Taurus.

17. Dévieux J, Malow R, Stein J, Jennings T, Lucenko B, AverHaRT $C$ et al. Impulsivity and HIV risk among adjudicated alcohol- and other drug-abusing adolescent offenders. AIDS Educ Prev 2004; 14, Supplement B: 24-35.

18. Lucenko B, Malow R, Sánchez-Martínez M. Negative affect and HIV risk in alcohol and other drug (AOD) abusing adolescent offenders. J Child Adolesc Subst Abuse 2003; 13: 1-17.

19. Malow R, Dévieux J, Jennings T. Substance-abusing adolescents at varying levels of HIV risk: Psychosocial characteristics, drug use, and sexual behaviour. J Subst Abuse 2001; 13: 103-17.

20. Grilo C, Sanislow C, Fehon D, Martino S, Mcglushan T. Psychological and behavioral functioning in adolescent psychiatric inpatients who report histories of childhood abuse. Am J Psychiatry 1999; 156: 538-44. 